\title{
Temperature distribution of micro milling process due to uncut chip thickness
}

\begin{abstract}
A simplified model for micro milling process is presented, as well as results on temperature on tool and work piece. The purpose is to investigate on finite element modelling of two flute micro end milling process of titanium alloy, Ti6Al4V with prediction of temperature distribution. ABAQUS/Explicit has been chosen as solver for the analysis. A thermomechanical analysis was performed. First model was created by selecting medium carbon steel, AISI1045, as workpiece material for model validation purpose. Second model was created by modifying the workpiece material from AISI1045 to Ti6Al4V. The model consists of two parts which are tungsten carbide micro tool and workpiece. Johnson-Cook law model has been applied as material constitutive properties for both materials due to its severe plastic deformation occur during machining. Prediction on forces was obtained during the analysis. Model validation was done by comparing results published by Woon et al. in 2008. The results showed a good agreement in cutting force. Once this was proved, the same model was then modified to simulate finite element analysis in micro milling of Ti6Al4V. Prediction of temperature distribution of micro end mill of Ti6Al4V was done in relation of different undeformed chip thickness. The findings showed that temperature increases as undeformed chip thickness increases. Temperature distribution of Ti6Al4V and AISI1045 under same machining conditions was compared. Results showed that the highest temperature was concentrated at tool edge for Ti6Al4V.
\end{abstract}

Keyword: Micro milling; Temperature distribution; Titanium 6-4; Uncut chip thickness 